Esta revista forma parte del acervo de la Biblioteca Jurídica Virtual del Instituto de Investigaciones Jurídicas de la UNAM

\title{
Indeterminación del estatus jurídico del migrante por cambio climático*
}

\author{
Indetermination of Legal Status of the Migrant \\ by Climate Change
}
Indetermination du statut juridique du migrant par le changement climatique

SUmario: I. Nota introductoria. II. Cifras que reflejan la problemática. III. Estatus jurídico, definiciones y categorías doctrinales. IV. El caso de Ioane Teitiota: ciudadano de Kiribati. V. Reflexión final. VI. Bibliografía.

* Estudio preliminar en el marco del proyecto en materia de Migraciones humanas y cambio climático. En proceso.

** Licenciada en derecho por la UNAM. Doctora en derecho ambiental por la Universidad de Alicante, España. Investigadora adscrita al área de derecho ambiental en el Instituto de Investigaciones Jurídicas de la UNAM.

Artículo recibido el 26 de junio de 2019

Aprobado para publicación el 3 de octubre de 2019 
RESUMEN: El presente trabajo destaca la importancia de la adopción de un concepto jurídico que reconozca al migrante por cambio climático en el ámbito del derecho internacional. Las migraciones poblacionales transfronterizas a causa de los impactos del cambio climático antropogénico son una realidad que requieren del reconocimiento, más que doctrinario, jurídico, en el ámbito del derecho internacional. La variedad de acepciones y categorías doctrinales (no vinculantes), reflejan la falta de enfoque del problema y los vacíos legales a afrontar. Actualmente no existe consenso sobre un concepto legal e unívoco que defina a las personas que se desplazan por cambio climático, su estatus jurídico es indeterminado, así como el instrumento normativo aplicable que proporcione la necesaria protección jurídica internacional, que les asegure a los migrantes una reubicación planificada fuera de sus países de origen y que garantice el respeto de sus derechos. Las consecuenias legales ante la falta de estatus jurídico son reflejadas al analizar el caso del señor Ioane Teitota, ciudadano de Kiribati, quien ante las Cortes de Nueva Zelanda solicitó el estatus de refugiado debido a las dificultades que enfrenta su país con respecto a los impactos del cambio climático, especialmente por la elevación del nivel del mar.

Palabras clave: migración, migrante, refugiado, desplazado, cambio climático, Kiribati.

ABSTRACT: This paper highlights the importance of the establishment of a legal term that recognizes the climate change displaced people in the framework of International Law. Transborder migrations caused by the impacts of anthropogenic climate change are a reality that require recognition, rather than doctrinal, legal, in the field of International Law. The variety of doctrinal meanings and categories (not legally binding) shows up the lack of focus of the problem and the legal gaps to face. Currently there is no consensus on a legal and univocal concept that defines people who are displaced by climate change, its legal status its undetermined, as well as the applicable normative instrument that provides the necessary international legal protection, which assures migrants a planned relocation outside their home countries and that guarantees respect for their rights. The legal consequences of the lack of legal status are reflected when analyzing the case of Kiribati's citizen, Mr. Ioane Teitota, who, before the New Zealand Courts, requested refugee status due to the difficulties that he faced at his country with respect to the impacts of climate change, particularly in the face of sea level rise.

Key words: Migration, Migrant, Refugee, Displaced, Climate Change, Kiribati.

RÉSUMÉ: Cet article souligne l'importance d'adopter un concept juridique qui reconnaît les migrants face au changement climatique dans le domaine du droit international. Les migrations transfrontalières de population dues aux impacts du changement climatique anthropique sont une réalité qui nécessite une reconnaissance, plutôt qu'une doctrine, un fondement juridique, dans le domaine du droit international. La variété de significations doctrinales et de catégories (non contraignantes) reflète le manque de focalisation du problème et les lacunes juridiques à combler. Il n'y a actuellement aucun consensus sur un concept juridique et univoque qui définit les personnes déplacées par le changement climatique, leur statut juridique est indéterminé, ainsi que sur l'instrument normatif applicable qui fournit la protection juridique internationale nécessaire, garantissant aux migrants une réinstallation planifiés en dehors de leur pays d'origine et garantissant le respect de leurs droits. Les conséquences juridiques de l'absence de statut juridique sont reflétées lors de l'analyse du cas de M. Ioane Teitota, citoyen de Kiribati, qui, devant les tribunaux néo-zélandais, a demandé le statut de réfugié en raison des difficultés rencontrées par son pays en ce qui concerne: Impacts du changement climatique, notamment en raison de l'élévation du niveau de la mer.

Mots-clés: migration, migrant, réfugié, déplacé, changement climatique, Kiribati 


\section{NOTA INTRODUCTORIA}

La relación entre las migraciones poblacionales transfronterizas e internas y el cambio climático inducido y acelerado por causa antrópica, es un asunto que cada vez toma más relevancia, requiriendo de especial atención en el marco jurídico internacional.

Newland ${ }^{1}$ señala que, básicamente son cuatro los efectos físicos del cambio climático que se vinculan con los desplazamientos masivos: el aumento del nivel del mar, el incremento de las temperaturas, los cambios en los ciclos hidrológicos y los ciclones tropicales cada vez más frecuentes e intensos, lo cual genera riesgos y provoca situaciones de emergencia.

La variabilidad climática y sus efectos en los medios de sustento, ${ }^{2}$ la salud, ${ }^{3}$ la disponibilidad de agua, ${ }^{4}$ entre otros, afectarán la calidad de vida y la vida misma, lo que propiciará migraciones humanas ${ }^{5}$ de igual modo, la elevación de los niveles del mar provocará que varias zonas costeras y los pequeños Estados insulares dejen de ser habitables; a su vez, la desertificación, la erosión, las inundaciones y sequías pueden generar movimientos de población. ${ }^{6}$

1 Newland, Kathleen, Climate Change and Migration Dynamics, Washington, Migration Policy Institute, 2011, pp. 4-7.

2 Véase, Ibarra, Rosalía, "Cambio climático, seguridad alimentaria y el derecho humano a la alimentación”, en Ibarra, Rosalía (coord.), Cambio climático y gobernanza. Una visión transdisciplinaria, México, UNAM, Instituto de Iinvestigaciones Jurídicas, 2019 (en prensa); Miraglia, M. et al., "Climate Change and Food Safety: An Emerging Issue with Special Focus on Europe", Food and Chemical Toxicology, núm. 47, 2009; FAO, Climate Change: Implications for Food Safety, Food and Agriculture Organization of the United Nation, 2008.

3 Véase, OPS, Proteger la salud frente al cambio climático: evaluación de la vulnerabilidad y la adaptación, Washington, USA, Organización Panamericana de la Salud, 2012.

4 Robles, Agustín y Garatuza, Jaime, "Cambio climático en México: impactos esperados en la disponibilidad del agua”, en Cruz, Xóchitl et al. (coords.), México ante la urgencia climática: ciencia, política y sociedad, México, UNAM, CRIM, CIICH, PICC, 2015, pp. 219-242; Sánchez, Ignacio et al., "Impacto de la variabilidad climática en la disponibilidad de agua para producción agrícola en México”, en Cruz, Xóchitl et al. (coords.), op. cit., pp. 243-271.

5 Orsi, Adriana, "Desplazados ambientales”, Futuros, vol. VI, núm. 20, 2008.

6 Véase OIM, Medioambiente, cambio climático y migración: perspectiva y actividades de la OIM, Ginebra, Suiza, Organización Internacional para las Migraciones, 2011. 
Sin embargo, esto no es una novedad, ya que la escasez de recursos naturales y la degradación ambiental han dado lugar a oleadas de emigrantes a lo largo de la historia $;^{7}$ la cuestión es que actualmente el cambio climático agrava los fenómenos naturales repentinos, así como la degradación ambiental paulatina, ocasionando un mayor desarraigo, pérdida de viviendas, deterioro de los medios de sustento y producción de las comunidades afec$\operatorname{tadas}^{8}$ (las más vulnerables y menos resilientes), lo que pone de manifiesto el alto coste humano y la profunda injusticia que supone el cambio climático.

Actualmente, por la elevación del nivel del mar el foco está puesto en los pequeños Estados insulares del Caribe y el Pacífico donde la existencia de islas y atolones de coral está amenazada, por lo que los impactos del cambio climático son un asunto de supervivencia de personas y naciones que urge ser atendido ante el vacío jurídico en la protección internacional, para enfrentar a las migraciones forzadas transfronterizas provocadas por el fenómeno climático.

\section{CIFRAS QUE REFLEJAN LA PROBLEMÁTICA}

El profesor Norman Myers, de la Universidad de Oxford, señaló que en 1995 los movimientos migratorios por causas ambientales totalizaron al menos 25 millones de personas procedentes, principalmente, de África, cifra que podría aumentar, pero no sólo en el continente africano sino a nivel mundial hasta alcanzar 200 millones de personas para 2050, como consecuencia del calentamiento global y la elevación del nivel del mar; ${ }^{9}$ cifra que

7 Boano, C. et. al., Environmentally Displaced People Understanding the Linkages between Environmental Change, Livelihoods and Forced Migration, Oxford, U. K., Refugee Studies Centre, Oxford Department of International Development, University of Oxford, 2008, p. 5.

8 Ibarra, Rosalía, "Cambio climático y conflictos socioambientales: migraciones forzadas”, en Carmona Lara, María del Carmen Aurora y Acuña Herández, Ana Laura, La Constitución y los derechos ambientales, México, UNAM, Instituto de Investigaciones Jurídicas, 2015, p. 68.

9 Myers, Norman, Enviromental Refugees: An Emergent Security Issue, 13th Meeting of the OSCE Economic Forum, Session III Environment and Migration, Organization for Security and Cooperation in Europe, Praga, 23-27 de mayo de 2005, p. 1. 
ha sido ampliamente aceptada y reconocida por el Intergovernmental Panel on Climate Change (IPCC). ${ }^{10}$

La recopilación de datos en este contexto no es tarea sencilla, amplio es el trabajo de la Oficina del Alto Comisionado de las Naciones Unidas para los Refugiados (ACNUR), el Centro de Monitoreo de Desplazamiento Interno - IDMC Internal Displacement Monitoring Centre-, ${ }^{11}$ y la Organización Internacional para las Migraciones (OIM), quienes continúan mejorando la disponibilidad y la cobertura de los datos mundiales sobre las poblaciones en situación de vulnerabilidad; cuyas dificultades metodológicas, operacionales y políticas surgen debido a la naturaleza de las situaciones de emergencia, así como a las características, la vulnerabilidad y la "visibilidad" de las poblaciones objeto de estudio. ${ }^{12}$

Sin embargo, de acuerdo con el Informe sobre las Migraciones en el Mundo 2010 de la OIM, ${ }^{13}$ los mejores datos disponibles que se pueden tener sobre los desplazados por motivos ambientales son "probablemente" los que se obtienen respecto a los movimientos poblacionales que se producen ante los fenómenos meteorológicos extremos, es decir, ante la degradación ambiental repentina.

En este contexto, es destacable la información del IDMC, aunque sólo se refiere a movimientos internos, mas no transfronterizos. En 2017, de acuerdo con el Global Report on Internal Displacement 2018, se registraron en 135 países, 18.8 millones de nuevos desplazamientos asociados con este tipo de eventos climatológicos; como en años anteriores las zonas con alto riesgo de desastre son Asia meridional, Asia oriental y el Pacífico, en cuanto a las Américas, éstas se vieron desproporcionadamente afectadas. Se destaca que la gran mayoría de los nuevos desplazamientos se desencadenaron por

10 Para más estimaciones véase Boano et al., op. cit., p. 12; Brown, Oli, 2008, "El baile de cifras”, Revista Migraciones Forzadas, núm. 31, 2008, pp. 8 y 9.

11 El IDMC a nivel mundial es la fuente autorizada de datos y análisis sobre el desplazamiento interno; fue establecido en 1998, como parte del Consejo Noruego para Refugiados —NRC Norwegian Refugee Council_-; proporcionan datos, análisis y experiencias mediante el monitoreo de situaciones de desplazamiento causadas por conflicto y violencia, desastres y desarrollo a nivel mundial.

12 OIM, Informe sobre las migracione en el mundo 2018, Ginebra, Organización Internacional para las Migraciones (OIM), 2018, p. 45.

13 OIM, Informe sobre las migraciones en el mundo 2010, El futuro de la migración: creación de capacidades para el cambio, Ginebra, Suiza, Organización Internacional para las Migraciones (OIM), 2010. 
inundaciones que representan 8.6 millones; y tormentas, principalmente ciclones tropicales (huracanes), con 7.5 millones; China, Filipinas, Cuba, Estados Unidos e India fueron los más afectados. Los huracanes Harvey, Irma y María rompieron varios registros en el Atlántico y el Caribe, y una serie de tifones en el sur y este de Asia y el Pacífico desplazó a gran número de personas en todo el año. ${ }^{14}$

En los registros del IDMC, son relevantes los datos señalados en el estudio: Displacement due to Natural Hazard-Induced Disasters Global Estimates for 2009 and 2010, en el cual se estimó que en 2010 más de 42 millones personas en todo el mundo se vieron obligadas a abandonar sus hogares debido a desastres desencadenados por peligros naturales repentinos (incluidos los terremotos); cabe apuntar que 38.3 millones, el 90\% del total, fueron desplazados debido a fenómenos relacionados con el clima (hidrológicos, meteorológicos o climatológicos) provocados principalmente por inundaciones y en algunos casos tormentas. Los eventos climatológicos como las temperaturas extremas y los incendios forestales tuvieron un impacto relativamente pequeño. ${ }^{15}$

En 2008, de los 36.5 millones de personas desplazadas por fenómenos naturales, 20 millones tuvieron como causa algún evento relacionado con el clima; ${ }^{16}$ mientras que en 2012, se vieron desplazadas 32.4 millones, o sea el 98\%, por inundaciones y tormentas. Estos tres años, 2010, 2008 y 2012 (en ese orden), cuentan con el mayor registro de desplazados internos por eventos climáticos; la zona que especialmente ha resultado afectada es Asia, seguida de África Occidental y Central. ${ }^{17}$

Las cifras señaladas desde luego muestran la relación entre la migración interna y el deterioro repentino generado por el cambio climático, no obs-

14 IDMC y NRC, Global Report on Internal Displacement GRID 2018, Ginebra, Switzerland, Internal Displacement Monitoring Centre (IDMC), Norwegian Refugee Council (NRC), 2018 , pp. 6 y 7.

15 IDMC y NRC, Displacement due to natural hazard-induced disasters, Ginebra, Internal Displacement Monitoring Centre (IDMC), Norwegian Refugee Council (NRC), 2011, pp. 11-13.

16 IDMC, NRC y UN OCHA, Monitoring Disaster Displacement in the Context of Climate Change, Ginebra, Internal Displacement Monitoring Centre (IDMC), Norwegian Refugee Council (NRC), United Nations Office for the Coordination of Humanitarian Affairs (UN OCHA), 2009, pp. 9-13 y 15.

17 IDMC y NRC, Global estimates 2012: People displaced by disasters, Ginebra, Internal Displacement Monitoring Centre (IDMC), Norwegian Refugee Council (NRC), 2013, pp. 6, 7 y $11-13$. 
tante, hasta ahora no se cuenta con una base de datos estadísticos respecto a los movimientos poblacionales transfronterizos por fenómenos meteorológicos extremos, así como por el deterioro ambiental paulatino. ${ }^{18}$

Al respecto, la OIM en su reciente Informe sobre las Migraciones en el Mundo 2018, reconoce que "una de las principales lagunas es la falta de datos cuantitativos sobre los movimientos asociados con el cambio ambiental, en particular con el cambio climático”, ya que son escasos los datos sobre las tendencias de movilidad a largo plazo asociadas con los desastres desencadenados por fenómenos naturales, así como la duración de los desplazamientos y trayectorias subsiguientes. ${ }^{19}$

\section{ESTATUS JURÍDICO, DEFINICIONES Y CATEGORÍAS DOCTRINALES}

En este apartado analizaremos que, mientras existe una amplia variedad de definiciones y categorías doctrinales para conceptualizar en términos generales a las personas que se desplazan por motivos ambientales y/o, particularmente, por los impactos del cambio climático; por el contrario, jurídicamente, no hay un reconocimiento legal que los defina y en consecuencia los proteja, ello especialmente en el ámbito internacional, ya que la degradación ambiental y/o los impactos del cambio climático no son estipulados para obtener el estatus de "refugiado" de conformidad con el instrumento internacional en la materia, el cual analizaremos enseguida.

\section{El régimen jurídico internacional de los refugiados}

Al finalizar la Segunda Guerra Mundial, en un contexto muy particular, el 28 julio de 1951 es adoptada la Convención de Ginebra sobre el Estatuto de los Refugiados, en la cual se señala en el artículo 1o., apartado A, 2), que el término refugiado es aplicable a toda persona que:

18 OIM, Informe sobre las Migraciones en el Mundo 2010. El futuro de la migración: creación de capacidades para el cambio, cit., p. 77.

19 OIM, Informe sobre las Migraciones en el Mundo 2018, cit., p. 45. 
... debido a fundados temores de ser perseguida por motivos de raza, religión, nacionalidad, pertenencia a determinado grupo social u opiniones políticas, se encuentre fuera del país de su nacionalidad y no pueda o, a causa de dichos temores, no quiera acogerse a la protección de tal país; o que, careciendo de nacionalidad y hallándose, a consecuencia de tales acontecimientos, fuera del país donde antes tuviera su residencia habitual, no pueda o, a causa de dichos temores, no quiera regresar a él.

De la definición anterior se desprende que adquiere el estatus de "refugiado" aquel que se ha desplazado por temor a una persecución, la cual debe estar motivada por cuestiones raciales, religiosas, políticas o sociales, dejando afuera cualquier otro factor que permita a un individuo o a un determinado grupo de personas calificarse como refugiados bajo este régimen internacional ${ }^{20}$ de tal manera que, quienes se ven obligados a abandonar su país debido al cambio climático, o que se van porque el cambio climático les dificulta ganarse el sustento en su país de origen, no pueden jurídicamente solicitar el estatus de refugiado conforme a la citada Convención; lo cual queda reforzado en el artículo 33, apartado 1, referente a la prohibición de expulsión y de devolución ("refoulement»), donde se establece explícitamente el alcance de la Convención, al señalar que: "Ningún Estado contratante podrá, por expulsión o devolución, poner en modo alguno a un refugiado en las fronteras de los territorios donde su vida o su libertad peligre por causa de su raza, religión, nacionalidad, pertenencia a determinado grupo social, o de sus opiniones políticas".

En el mismo sentido, la Convención de la Organización de la Unidad Africana por la que se regulan los aspectos específicos de problemas de los refugiados en África, aprobada el 10 de septiembre de 1969, además de contemplar la definición de la Convención sobre el Estatuto de los Refugiados, establece en el párrafo 2 del artículo 1o. que:

El término "refugiado" se aplicará también a toda persona que, a causa de una agresión exterior, una ocupación o una dominación extranjera, o de acontecimientos que perturben gravemente el orden público en una parte o en la totalidad de su país de origen, o del país de su nacionalidad, está obligada a abandonar su residen-

20 Ibarra, Rosalía, "Cambio climático y conflictos socioambientales: migraciones forzadas", cit., p. 74. 
Esta revista forma parte del acervo de la Biblioteca Jurídica Virtual del Instituto de Investigaciones Jurídicas de la UNAM

DOI: http://dx_doi.org/10.22201/iij_24487872e_2020.20.14473

cia habitual para buscar refugio en otro lugar fuera de su país de origen o del país de su nacionalidad.

A su vez, la Declaración de Cartagena sobre Refugiados, adoptada el 22 de noviembre de 1984, establece que en América Central, México y Panamá, la definición o concepto de refugiado recomendable es:

... aquella que además de contener los elementos de la Convención de 1951 y el Protocolo de 1967, considere también como refugiados a las personas que han huido de sus países porque su vida, seguridad o libertad han sido amenazadas por la violencia generalizada, la agresión extranjera, los conflictos internos, la violación masiva de los derechos humanos u otras circunstancias que hayan perturbado gravemente el orden público.

Por lo tanto, los refugiados son personas que huyen de conflictos armados o de persecución, su situación es tan peligrosa e intolerable, que cruzan fronteras nacionales para buscar seguridad en países cercanos, y así, ser reconocidos internacionalmente como "refugiados", con asistencia de los Estados, el Alto Comisionado de las Naciones Unidas para los Refugiados (ACNUR) y otras organizaciones; su reconocimiento es a razón de que para ellos es demasiado peligroso regresar a casa, y necesitan asilo en otros lugares con el fin de evitar consecuencias mortales. De tal manera que la protección de los refugiados implica la protección ante los peligros de los cuales han huido, el acceso a procedimientos de asilo y medidas que garanticen que sus derechos humanos sean respetados, en lo que se encuentra una solución a largo plazo. Los países legalmente aplican el marco jurídico en materia de asilo y la protección de los refugiados. ${ }^{21}$

No obstante, la realidad actual impone nuevas y diversas causas que motivan desplazamientos involuntarios, como las sequías, la desertificación, la deforestación, las inundaciones, la degradación del suelo, etcétera; de ahí la inminente necesidad de adoptar o actualizar disposiciones jurídicas para el reconocimiento y protección de las migraciones climáticas.

21 Edwards, Adrian, “¿«Refugiado» o «migrante»? ¿Cuál es el término correcto? Los dos términos tienen diferentes significados, y confundirlos conduce a problemas para ambas poblaciones”, UNHCR. ACNUR La Agencia de la ONU para los Refugiados, 11 de julio, 2016 www. acnur.org/noticias/noticia/2016/7/5b9008e74/refugiado-o-migrante-cual-es-el-termino-correcto. html. 
En este sentido, es interesante la postura de Biermann y Boas, ${ }^{22}$ quienes argumentan que no hay una razón convincente para reservar el término de refugiado para una categoría de personas quienes se hicieron el centro de atención, después de 1945, pues al día de hoy existen nuevas categorías de personas que se ven forzadas a abandonar sus hogares con consecuencias igualmente graves; además, consideran que el término "refugiado" tiene fuertes connotaciones morales de protección societal en la mayoría de las culturas y las religiones, por lo que emplear este término para los desplazados climáticos les proporcionará legitimidad y la urgente protección que merecen.

\section{El desplazado interno}

Las migraciones forzadas dentro de un mismo país por causas vinculadas con el cambio climático se entienden reconocidas en los Principios Rectores de los Desplazamientos Internos de 1998, ello al establecer que los desplazados internos son:

... las personas o grupos de personas que se han visto forzadas u obligadas a escapar o huir de su hogar o de su lugar de residencia habitual, en particular como resultado o para evitar los efectos de un conflicto armado, de situaciones de violencia generalizada, de violaciones de los derechos humanos o de catástrofes naturales o provocadas por el ser humano, y que no han cruzado una frontera estatal internacionalmente reconocida. ${ }^{23}$

Personas a quienes, las autoridades nacionales deben proporcionarles asistencia humanitaria y protección, ${ }^{24}$ la cual debe comprender acciones que: $:^{25}$

22 Biermann, Frank y Boas, Ingrid, "Climate Change and Human Migration. Towards a Global Governance System to Protect Climate Refugees”, en Scheffran, J. et al. (eds.), Climate Change, Human Security and Violent Conflict, Berlin, Springer-Verlag, 2012, p. 292.

23 Numeral 2 del apartado de "Introducción: alcance y finalidad".

24 Principio 3, numeral 1, "Principios rectores de los desplazamientos internos".

25 Droege, Cordula, "Progresos en la protección jurídica de los desplazados internos", Revista Migraciones Forzadas GP 10, diciembre, 2018, p. 9. 
1) Prevengan o erradiquen un patrón concreto de abuso o atenúe sus efectos inmediatos.

2) Devuelvan la dignidad a las personas y garanticen condiciones de vida adecuadas.

3) Promuevan un entorno que propicie el respeto de los derechos humanos de conformidad con la normativa pertinente.

En este sentido, las soluciones deben ser duraderas y a largo plazo, sobre todo ante el rasgo significativo que se desprende de la definición arriba señalada, y que es la involuntariedad individual o colectiva al no tener otra elección ante conflictos armados, disturbios o catástrofes naturales u ocasionadas por el hombre, ${ }^{26}$ tal es el caso del cambio climático antropogénico, no obstante en los principios citados no se hace una mención expresa sobre el vínculo entre la migración forzada y el cambio climático, esto más bien se entiende de manera tácita.

A su vez, es relevante lo establecido en la Convención de la Unión Africana para la Protección y la Asistencia de los Desplazados Internos en África (Convención de Kampala), adoptada el 22 de octubre de 2009, con entrada en vigor el 6 de diciembre de 2012, la cual, además de definir en el artículo 1o., inciso $\mathrm{k}$, a los desplazados internos en los términos arriba expuestos, establece en el artículo 5o. la amplia obligación estatal de brindar protección y asistencia conforme a los siguientes puntos, siendo de especial importancia el cuarto:

1) En los Estados parte recae el deber primordial y la responsabilidad de brindar protección y asistencia humanitaria, sin discriminación de ningún tipo, a los desplazados internos, que se encuentren en su territorio o jurisdicción.

2) Los Estados parte cooperarán entre sí, a petición del Estado parte interesado o de la Conferencia de Estados parte, en la protección y asistencia a los desplazados internos.

3) Los Estados parte respetarán los mandatos de la Unión Africana y las Naciones Unidas, así como las funciones de las organizaciones huma-

26 Cfr. "Forced Displacement y Forced Migration", en IOM, Glossary on Migration, 2a. ed., International Migration Law, núm. 25, Ginebra, International Organization for Migration, 2011, p. 39. 
Esta revista forma parte del acervo de la Biblioteca Jurídica Virtual del Instituto de Investigaciones Jurídicas de la UNAM

nitarias internacionales en la prestación de protección y asistencia a los desplazados internos, de conformidad con el derecho internacional.

4) Los Estados parte adoptarán medidas para proteger y ayudar a las personas que han sido desplazadas internamente debido a los desastres naturales o producidos por el ser humano, incluyendo el cambio climático. ${ }^{27}$

5) Los Estados parte deberán evaluar o facilitar la evaluación de las necesidades y vulnerabilidades de los desplazados internos y de las comunidades de acogida, en cooperación con las organizaciones o agencias internacionales.

6) Los Estados parte facilitarán suficiente protección y asistencia a los desplazados internos, y cuando los recursos disponibles sean insuficientes para ello, cooperarán en la búsqueda de la asistencia de organizaciones internacionales y organismos humanitarios, organizaciones de la sociedad civil y otros actores relevantes. Dichas organizaciones pueden ofrecer sus servicios a todos que los que necesiten...

La Convención de Kampala basada en los Principios Rectores de los Desplazamientos Internos es el primer marco regional común que define los roles y responsabilidades de una amplia gama de instituciones y organizaciones que operan en situaciones de desplazamiento $;^{28}$ sin duda, la Convención representa un avance importante en la creación de un sistema regional de protección y asistencia para los desplazados internos al establecer las obligaciones de los Estados y reconocer al cambio climático como una causa del desplazamiento. El secretario general de la ONU ha destacado que la Convención sirve de modelo en los proyectos regionales similares que promuevan la adopción y aplicación de marcos jurídicos y políticos regionales sobre desplazamiento interno. ${ }^{29}$

27 Énfasis añadido.

28 Global Protection Cluster, Marcos normativos sobre desplazamiento interno. Desarrollos mundiales, regionales y nacionales, The UN Refugee Agency (UNHCR), Internal Displacement Monitoring Centre (IDMC), Norwegian Refugee Council (NRC), 2016, p. 11.

29 Ibidem, p. 17. 


\section{Refugiados ambientales}

En 1985 en el Informe Environmental Refugees, Essam El-Hinnawi definió el término de "refugiado ambiental" como:

Aquellas personas que han sido forzadas a abandonar su hábitat tradicional de manera temporal o permanente, debido a una marcada perturbación ambiental (natural y/o desencadenada por las personas) que ha puesto en peligro su existencia y/o ha afectado gravemente su calidad de vida. Por "perturbación ambiental" se entiende cualquier cambio físico, químico o biológico en el ecosistema (o sus recursos base) que lo transforma en no apto para la vida humana, ya sea temporal o permanentemente. ${ }^{30}$

De lo anterior se desprenden tres categorías de "refugiados ambientales": 31

1) Los temporales a causa de un evento natural inesperado, y con la probabilidad de regresar a su hábitat original. En este caso estaríamos frente al deterioro ambiental repentino.

2) Los permanentes, debido a los cambios en su hábitat causados por la actividad humana, como accidentes industriales o la ejecución de mega proyectos de desarrollo. En este caso, Soledad ${ }^{32}$ los coloca en la categoría de "desplazados inducidos por el desarrollo", es decir, aquellos afectados "directamente por grandes proyectos de infraestructura como embalses, carreteras, urbanizaciones, nuevos regadíos, medidas urbanas higienistas, adquisiciones y expropiaciones arbitrarias de tierra”.

3) Los permanentes en busca de una mejor calidad de vida, debido a que en su hábitat original ya no puede satisfacer sus necesidades básicas; lo que hace referencia al deterioro ambiental paulatino; como es el caso

30 El-Hinnawi, Essam, Environmental Refugees, Nairobi, United Nations Environment Programme (UNEP), 1985, p. 4. Traducción propia.

31 Idem; Lonergan, Steve, "The Role of Environmental Degradation in Population Displacement", Environmental Change and Security Program Report, Issue 4, spring, 1998, pp. 7 y 8.

32 Soledad, Javier Iván, Desplazamiento interno en Colombia. La situación de los desplazados en El Catatumbo, España, Editorial Universidad de Granada, 2009, 102. 
de los agricultores que abandonan sus tierras a causa de la degradación de los suelos.

Por lo tanto, los refugiados ambientales son aquellas personas que han sido forzadas a dejar su hábitat tradicional, de forma temporal o permanente, debido a un deterioro ambiental, ya sea causado de manera natural y/o provocado por la actividad humana, como accidentes industriales, la realización de grandes proyectos económicos, la mala gestión de residuos tóxicos, etcétera, lo que provoca en estos últimos casos un desplazamiento permanente, al verse amenazada la calidad de vida, la salud y en sí la vida misma. ${ }^{33}$

Por su parte el catedrático Norman Myers define a los refugiados ambientales como:

Personas que ya no pueden ganarse la vida en sus países de origen a causa de la sequía, la erosión del suelo, la desertificación, la deforestación y otros problemas ambientales, junto con problemas asociados de las presiones demográficas y la pobreza profunda. En su desesperación, estas personas sienten que no tienen otra alternativa sino buscar refugio en otro lugar, cuyo intento es peligroso. No todos han huido de sus países, muchos se desplazan internamente, otros por el contrario han abandonado sus países de origen de forma semipermanente, o permanente, con pocas esperanzas de un retorno inmediato. ${ }^{34}$

En importante señalar que en las definiciones anteriores se destaca la degradación ambiental inducida por las actividades humanas, pues hay que entender que en el caso que nos ocupa, los movimientos migratorios no se generan por "culpa" de la naturaleza en su estado simple y llano, sino más bien, es a causa del desarrollo insostenible que perturba, acelera y agrava los fenómenos naturales, y que, al día de hoy, nos ha colocado ante el más grande problema ambiental global: el cambio climático por causas antropogénicas con graves consecuencias sociales.

33 Véase Borràs, Susana, "Refugiados ambientales: el nuevo desafío del derecho internacional del medio ambiente”, cit., p. 89.

34 Myers, Norman, Enviromental Refugees: An Emergent Security Issue, cit., pp. 6 y 7. Traducción propia. 
Finalmente, no podemos dejar de señalar la definición que, contrario a lo expuesto líneas arriba, la División de Estadística del Departamento de Información, Económica y Social y Análisis de Políticas de las Naciones Unidas, pronuncia de una manera muy simple, restrictiva y con un lenguaje erróneo, pues define al refugiado ambiental en el Glosario de Estadísticas del Medio Ambiente, como aquella persona que ha sido "desplazada debido a causas ecológicas, principalmente la pérdida y degradación de tierras, y los desastres naturales. También se denomina refugiado a causa de problemas ambientales". ${ }^{35}$

En principio, habría que señalar que más que ecológicas son ambientales, sin embargo, cabe apuntar que en la versión en inglés acertadamente se redactó "environmental causes"; ${ }^{36}$ segundo, las causas están limitadas al señalar de manera principal sólo la degradación de suelos; tercer aspecto, equivocadamente se habla de desastres naturales, cuando la naturaleza, en sí misma no es un desastre y no causa desastres, lo que existe son fenómenos naturales, el desastre es social, como consecuencia de la gestión errónea de los recursos naturales por parte de la especie humana, al destruir los ecosistemas, y sobre todo al desafiar el curso natural de los fenómenos naturales, y más aún cuando tenemos amplio conocimiento de sus consecuencias. En sí, esta definición justamente no alude a la degradación ambiental antropogénica.

Por otro lado, autores como Black ${ }^{37}$ niegan la existencia del concepto de refugiado ambiental, al sostener que la degradación ambiental puede ser un factor importante en la decisión de emigrar; sin embargo, establecer un concepto en donde sea la causa principal lo considera sin fundamentos sólidos, y un vínculo que aún está por probar; postura que resulta un tanto escéptica.

35 ONU, Glosario de Estadísticas del Medio Ambiente, Nueva York, Naciones Unidas, División de Estadística, Departamento de Información, Económica y Social y Análisis de Políticas, 1997, p. 97.

36 Véase "Environmental Refugee”, en ONU, Glossary of Environment Statistics, New York, United Nations, Statistics Division, Department for Economic and Social Information and Policy Analysis, 1997, p. 30, disponible en: http://unstats.un.org/unsd/environmentgl/gesform. asp? getitem $=473$.

37 Black, Richard, "Environmental Refugees: Myth or Reality?", New Issues in Refugee Research,Working Paper 34, UNHCR, 2001, p. 3. 


\section{Migrantes ambientales}

Es importante señalar que, a nivel internacional, no existe una definición legal para el migrante; no obstante, las Naciones Unidas lo define como:

Un individuo que ha residido en un país extranjero por más de un año, independientemente de las causas, voluntarias o involuntarias, y los medios, regulares o irregulares, utilizados para migrar. Bajo tal definición, aquellos que viajan por periodos más cortos como turistas y empresarios no serían considerados migrantes. Sin embargo, el uso común incluye ciertos tipos de migrantes a corto plazo, como los trabajadores agrícolas temporales que viajan por periodos cortos para trabajar en la siembra o cosecha de productos agrícolas. ${ }^{38}$

Los migrantes no son personas que huyen de la guerra o la persecución en su país de origen y que, por tanto, deben cruzar fronteras, los migrantes, a diferencia de los refugiados, pueden volver a su país de forma segura y continúan recibiendo la protección de su gobierno, por lo que son tratados de conformidad con su propia legislación y procedimientos en materia de inmigración. Por lo tanto, el migrante se refiere "a personas que se trasladan por razones no incluidas en la definición legal de refugiado". ${ }^{39}$

Por su parte, la palabra migración se refiere a:

El movimiento de una persona o un grupo de personas, ya sea a través de una frontera internacional o dentro de un Estado. Es un movimiento de población, que abarca cualquier tipo de movimiento de personas, cualquiera que sea su duración, composición y causas; incluye la migración de refugiados, personas desplazadas, migrantes económicos y personas que se desplazan para otros fines, incluida la reunificación familiar. ${ }^{40}$

En este contexto, la Organización Internacional para las Migraciones define a los "migrantes por motivos ambientales", sin que ello tenga repercusiones de carácter normativo, como:

38 IOM, Glossary on Migration, cit., p. 62. Traducción propia.

39 Edwards, Adrian, “¿«Refugiado» o «Migrante»? ¿Cuál es el término correcto? Los dos términos tienen diferentes significados, y confundirlos conduce a problemas para ambas poblaciones", cit.

40 IOM, Glossary on Migration, cit., pp. 62 y 63. Traducción propia. 
DOI: http://dx_doi.org/10.22201/iij_24.487872e_2020.20.14473

[Aquellas] personas o grupos de personas que, por razones insoslayables de cambios repentinos o progresivos en el medio ambiente que afectan de forma negativa a su supervivencia o a sus condiciones de vida, se ven obligadas a abandonar su hogar habitual, o deciden hacerlo, ya sea temporal o permanentemente, y se desplazan o bien dentro de su país o bien al extranjero. ${ }^{41}$

En esta definición, la OIM no hace una distinción entre la degradación ambiental natural y la inducida, sin embargo, al igual que en las definiciones de los "refugiados ambientales", se hace referencia al carácter temporal o permanente, así como a la migración interna y transfronteriza; por otro lado, resulta destacable el factor voluntario y obligatorio, lo cual nos lleva a plantear la migración voluntaria y la forzada por motivos ambientales.

Al respecto, Graeme señala que la movilidad de la población (en el contexto del cambio ambiental) probablemente ha de considerarse un fenómeno que ocurre a lo largo de un proceso que abarca desde la migración totalmente voluntaria a la migración totalmente forzosa; ${ }^{42}$ esta última entendida como un movimiento migratorio en el que existe un elemento de coacción, incluidas las amenazas a la vida y al sustento, ya sea por causas naturales o provocadas por el hombre, por ejemplo, movimientos de refugiados y de desplazados internos, además de personas desplazadas por desastres naturales o ambientales, desastres químicos o nucleares, hambruna o proyectos de desarrollo. ${ }^{43}$

De acuerdo con Egea y Soledad, ${ }^{44}$ la diferencia entre la migración voluntaria y la forzada radica en un factor temporal, al señalar que, en las primeras se planifica la salida según circunstancias y escenarios diversos, mientras que en las segundas no existe tiempo disponible para planificar o diseñar el "proyecto migratorio", constituyendo, una huida, la salida en más de una ocasión.

41 OIM, Diálogo Internacional sobre la Migración. Seminario de Expertos: Migración y Medio Ambiente, núm. 10, Ginebra, Organización Internacional para las Migraciones y Programa de Políticas e Investigación sobre Migraciones, 2008, p. 23.

42 Graeme, Hugo, "Environmental Concerns and International Migration”, The International Migration Review, vol. 30, no. 1, Special Issue: Ethics, Migration, and Global Stewardship, primavera de 1996, p. 107.

43 IOM, Glossary on Migration, cit., p. 39. Traducción propia.

44 Egea, Carmen y Soledad, Javier Iván, "Los desplazados ambientales, más allá del cambio climático. Un debate abierto”, Cuadernos Geográficos, núm. 49, 2011, p. 201. 
En este contexto, en el plano ambiental, las migraciones voluntarias están ligadas a la degradación ambiental paulatina, mientras que las forzadas se vinculan con la degradación ambiental repentina, como lo es la derivada por ciclones tropicales; sin embargo, en ambos casos no existe una decisión voluntaria de fondo, es decir la propia migración por degradación ambiental paulatina es forzada, en donde efectivamente la diferencia estriba en un aspecto temporal que permite una planificación, pero el movimiento migratorio se genera obligatoriamente tarde o temprano debido a las dificultades de supervivencia como resultado de la destrucción, sobreexplotación y escasez de recursos naturales en el hábitat de las comunidades, lo cual "obliga" al desplazamiento poblacional.

$\mathrm{Al}$ respecto, la Asociación Internacional para el Estudio de la Migración Forzada $^{45}$ considera a los desplazados inducidos por desastres, dentro de cuya categoría se incluyen como causas a los desastres naturales (fenómenos naturales extremos: inundaciones, actividad volcánica, derrumbes, terremotos), el cambio ambiental (deforestación, desertificación, degradación de suelos y calentamiento global), y los desastres producidos por la actividad humana (accidentes industriales, radioactividad). Cabe apuntar que la Asociación distingue a las personas desplazadas por motivos ambientales de entre los otros tipos de migrantes forzados, como refugiados, solicitantes de asilo, personas internamente desplazadas, personas desplazadas por desarrollo, trata de personas y tráfico de personas. ${ }^{46}$

Por otro lado, Richmond destaca otro factor temporal a tomar en cuenta en la definición del migrante por motivos ambientales, al considerar que, independientemente de la necesidad de proveerles de ayuda ante la pérdida de sus medios de vida u hogares, si las personas desplazadas son capaces de volver a la zona de origen en un plazo de días, semanas o incluso meses, no pueden considerase como migrantes ambientales y menos aún como refugiados ambientales. ${ }^{47}$

45 International Association for the Study of Forced Migration (IASFM)

46 FMO, What is Forced Migration?, disponible en: http://www.forcedmigration.org/about/ whatisfm, 2012.

47 Richmond, A. H., "The Environment and Refugees: Theoretical and Policy Issues", Population Bulletin of the United Nations, no. 39, Nueva York, United Nations, 1995, p. 3. 


\section{Desplazados ambientales}

La Organización Internacional para las Migraciones define a las personas desplazadas por motivos ambientales como: Aquellas personas que se desplazan dentro de su país de residencia habitual o que han cruzado una frontera internacional y para quienes la degradación, deterioro o destrucción del medio ambiente es la causa principal, pero no la única de su desplazamiento. Este término se usa como una alternativa menos controvertida a la de refugiado ambiental o de refugiado climático que, no tienen base jurídica o razón de ser en el derecho internacional, para referirse a una categoría de migrantes por motivos del medio ambiente cuyo movimiento es de naturaleza claramente forzosa ${ }^{48}$ sobre todo en caso de quienes se han desplazado a través de una frontera internacional.

En esta definición se destaca el desplazamiento interno, así como el transfronterizo y, como se aprecia, las causas ambientales naturales e inducidas por la actividad humana están presentes al emplear los calificativos: degradación, deterioro o destrucción ambiental.

$\mathrm{Al}$ igual que en las diferentes definiciones sobre los "refugiados ambientales", podemos estimar que, de alguna manera, se alude a los efectos del cambio climático en el campo migratorio, al hacer referencia a la degradación ambiental repentina y paulatina, así como a la influencia humana.

\section{Otros conceptos y expresiones}

Las advertencias sobre los impactos del cambio climático en el desplazamiento poblacional han propiciado el uso de otros términos y expresiones como "migración inducida ambientalmente (MIA)", 49 "migración inducida por el cambio climático", "migrantes debido al cambio climático", "refugiados ecológicos", "migrantes forzados por motivos medioambientales", 50

48 IOM, Glossary on Migration, cit., p. 34. Traducción propia.

49 Oswald-Spring, Úrsula et al., Vulnerabilidad social y género entre migrantes ambientales, México, Cuernavaca, UNAM, Centro Regional de Investigaciones Multidisciplinarias, 2014, pp. 15 y ss.

50 Dun, Olivia y Gemenne, François, "Definir la migración por motivos medioambientales”, Revista Migraciones Forzadas, núm. 31, noviembre, 2008, p. 10. 
"migrante climático", "refugiado climático", ${ }^{1}$ "migrante forzoso por motivos climáticos". ${ }^{52}$

Incluso se ha empleado la expresión "climigración"; al respecto, Bronen señala que ésta se da "cuando una comunidad ya no puede mantenerse únicamente a causa de acontecimientos climáticos, por lo que se debe reubicar de forma permanente para proteger a sus miembros", ${ }^{53}$ asimismo establece tres elementos clave:

1) la continuidad de los acontecimientos climáticos;

2) el daño reiterado que éstos conllevan para las infraestructuras públicas, $\mathrm{y}$

3) la amenaza que suponen para la seguridad de las personas (amenaza que incluye el riesgo de perder vidas). ${ }^{54}$

No menos innovador es el término "ecomigración” empleado por Woods ${ }^{55}$ para hacer referencia al vínculo entre los cambios ambientales y la migración, quien señala que los "ecomigrantes" se desplazan de manera voluntaria en busca de recursos naturales, o bien de un modo forzado cuando los recursos se degradan o destruyen. Para este autor las causas de la "ecomigración” son tres: los desastres naturales, los desastres urbano-industriales, y la explotación y degradación de los recursos naturales; sin embargo, también apunta que este fenómeno migratorio se debe entender dentro del contexto de los vínculos socioeconómicos, culturales, políticos y ambientales entre los lugares de origen y destino.

A pesar del esfuerzo por definir a los movimientos migratorios generados por la degradación ambiental, o bien debido a los efectos del cambio climático, no existe consenso en la adopción de una definición o categoría, ni

51 Biermann, Frank y Boas, Ingrid, "Climate Change and Human Migration. Towards a Global Governance System to Protect Climate Refugees”, cit., 2012, pp. 291-300.

52 OIM, Migración y cambio climático, Ginebra, Suiza, Organización Internacional para las Migraciones, Serie de Estudios de la OIM sobre la Migración, núm. 31, 2008, p. 15.

53 Bronen, Robin, "Las comunidades de Alaska: derechos y resistencia”, Revista Migraciones Forzadas, núm. 31, noviembre, 2008, p. 31.

54 Idem.

55 Woods, William B., "Ecomigration. Linkages Between Environmental Change and Migration”, en Zolberg, Aristide R. y Benda, Peter M. (eds.), Global Migrants Global Refugees. Problems and Solutions, Nueva York-Oxford, Berghahn Books, 2001, pp. 42-61. 
tampoco respecto a un marco jurídico internacional que brinde protección a las personas que se desplazan por motivos ambientales y/o climáticos, ${ }^{56}$ lo cual sería fundamental para el desarrollo de políticas públicas que respondan a tal problemática, especialmente cuando se trata de migraciones transfronterizas.

No obstante, de acuerdo con Dun y Gemenne ${ }^{57}$ el principal motivo de la falta de definición o categoría reconocida jurídicamente está vinculado a la dificultad de aislar los factores ambientales de otros factores causantes de la migración; situación que se presenta especialmente cuando se trata de una degradación ambiental paulatina como la desertificación, donde las personas se adaptan u "optan" por emigrar, y cuyo reconocimiento como migrante o refugiado no resulta tan evidente.

A pesar de lo anterior, quienes socialmente están siendo afectados por los impactos del cambio climático, no les importa la definición o categoría doctrinal que sea adoptada, ni si existen nuevas conceptualizaciones, les importa la protección legal, que sigue sin ser estipulada en los instrumentos jurídicos internacionales ante migraciones transfronterizas, les importa un concepto jurídico que los reconozca, que les otorgue un estatus vinculante, eso es lo trascendente, tal cual se desprende del estudio del caso que, a continuación se expone, en donde el vínculo de migración por factores del cambio climático nunca se puso en duda por parte de los tribunales.

\section{El CASO DE IOANE TEITIOTA: CIUDADANO DE KiRIBATI}

Kiribati es un archipiélago y país insular ubicado en el Océano Pacífico al noroeste de Australia, con un poco más de 110,000 habitantes. El país está formado por 32 atolones de origen coralino y una elevada isla de coral llamada Banaba, dispersa en más de 3,500,000 km². Kiribati es un conjunto de islas separadas en tres grupos: las islas Gilbert, las islas Line y las islas Phoenix, con una superficie total de $811 \mathrm{~km}^{2}$; el terreno es en su mayoría

56 OIM, Informe sobre las Migraciones en el Mundo 2010, El futuro de la migración: creación de capacidades para el cambio, cit., p. 77.

57 Crf. Dun, Olivia y Gemenne, François, "Definir la migración por motivos medioambientales", cit., p. 10. 
atolones de coral bajos, rodeados por extensos arrecifes, con un área costera de $1,143 \mathrm{~km}$ de largo. ${ }^{58}$ Es un país fuertemente expuesto a los impactos del cambio climático, cuyo factor principal es la supervivencia ante la elevación del nivel del mar, ${ }^{59}$ lo cual se debe a que geográficamente la escasa elevación sobre el nivel del mar de muchas de las islas que integran este país no supera los tres metros, lo que las hace sumamente vulnerables ante el crecimiento de la marea. ${ }^{60}$ Datos satelitales indican que el nivel del mar ha aumentado en Kiribati entre 1 y 4 mm por año desde 1993, en comparación con el promedio mundial de 2.8 a $3.6 \mathrm{~mm}$ por año; y se proyecta para 2030 un aumento en el rango de 5 a $14 \mathrm{~cm}$, ello en un escenario con un alto porcentaje de emisiones de gases de efecto invernadero. ${ }^{61}$

Debido a estas circunstancias, Kiribati es un país que se está sumergiendo poco a poco y está en riesgo de desaparecer, con el inminente impacto social que ello conlleva, de ahí que la migración transfronteriza, e indudablemente forzada, a otro territorio sea planteada como una vía para enfrentar tal situación, sin embargo, como ha quedado señalado en el apartado anterior, ante las posibles migraciones a través de fronteras internacionales motivadas por los impactos del cambio climático, en el derecho internacional no está legalmente reconocido un estatus para su protección.

Lo anterior se constata ante el rechazo de otorgar el estatus de refugiados a familias de Kiribati que están sufriendo los efectos del cambio climático, ello bajo el argumento de que no hay una base legal, tal es el caso del señor Ioane Teitiota ante la Suprema Corte de Nueva Zelanda.

58 V'ease “About Kiribati”, disponible en: http://www.climate.gov.ki/about-kiribati/; Environment and Conservation Division, Ministry of Environment, Land, and Agricultural Development Government of Kiribati Republic of Kiribati. National Adaptation Programme of Action, 2007, pp. 1-5.

59 IPCC, "Resumen para responsables de políticas", en Field, C. B., et al. (eds.), Cambio climático 2014: impactos, adaptación y vulnerabilidad. Contribución del Grupo de trabajo II al Quinto Informe de Evaluación del Grupo Intergubernamental de Expertos sobre el Cambio Climático, Ginebra, Organización Meteorológica Mundial, 2014, pp. 11-25.

60 V'éase Kelly, Wyett, "Escaping a Rising Tide: Sea Level Rise and Migration in Kiribati”, Asian \& The Pacific Policy Studies, vol. 1, issue 1, 2013; Ministry of Internal \& Social Affairs, 6. South Tarawa, Republic of Kiribati Island Report Series, Office of Te Beretitenti and T'Makei Services, 2012, disponible en: http://www.climate.gov.ki/wp-content/uploads/2013/01/6_ SOUTH-TARAWA-revised-2012.pdf; AF (Kiribati) [2013] NZIPT 800413, párr. 13.

61 "Changing Climate or Kiribati", disponible en: http://www.climate.gov.ki/changingclimate/; Future climate of Kiribati, disponible en: http: / /www.climate.gov.ki/changing-climate/. 


\section{Ioane Teitiota v. The Chief Executive of the Ministry of Business, Innovation and Employment ${ }^{62}$}

El señor Ioane Teitiota y su esposa llegaron a Nueva Zelanda desde Kiribati en 2007; en ese entonces, él contaba con un visado para trabajar en la construcción por tres años, el cual expiró el 7 de octubre de 2010, a partir de ese momento permanecieron de manera ilegal. Tuvieron en Nueva Zelanda tres hijos; no obstante, por disposiciones legales de la Citizenship Act 1977 ninguno tiene derecho a la ciudadanía neozelandesa.

En 2011, después de ser detenido el señor Teitiota en una parada de tráfico, solicitó se le otorgara el estatus de refugiado de conformidad con la sección 129, y/o el estatus de persona protegida de acuerdo con la sección $131,{ }^{63}$ ambas de la Immigration Act $2009 .{ }^{64}$

De especial relevancia son los argumentos de la solicitud, fundamentados en la vulnerabilidad de su tierra natal por el aumento constante del nivel del mar como consecuencia del cambio climático, razón por la cual, los ciudadanos viven con el temor de que con el paso del tiempo, el progresivo aumento del nivel del mar y la degradación ambiental asociada los obligue

62 AF (Kiribati) [2013] NZIPT 800413; Ioane Teitiota vs. The Chief Executive of the Ministry of Business, Innovation and Employment [2014] NZCA 173; Ioane Teitiota vs. The Chief Executive of the Ministry of Business, Innovation and Employment, [2015] NZSC 107, New Zealand, Supreme Court, 20 July 2015.

63 Section 129(1) establece que: "A person must be recognised as a refugee in accordance with this Act if he or she is a refugee within the meaning of the Refugee Convention".

Section 131 (1) establece que: "A person must be recognised as a protected person in New Zealand under the Covenant on Civil and Political Rights if there are substantial grounds for believing that he or she would be in danger of being subjected to arbitrary deprivation of life or cruel treatment if deported from New Zealand

(6) In this section, cruel treatment means cruel, inhuman, or degrading treatment or punishment".

64 Asimismo, solicitó se le reconociera como persona protegida en virtud de la Convención contra la Tortura de 1984, según la sección 130(1) de la Inmigration Act 2009: "Section 130(1) of the Act provides that: A person must be recognised as a protected person in New Zealand under the Convention Against Torture if there are substantial grounds for believing that he or she would be in danger of being subjected to torture if deported from New Zealand".

Al respecto, cabe señalar que resultó improcedente el otorgamiento del estatus solicitado. Consúltese AF (Kiribati) [2013] NZIPT 800413, párrafos 77 y 78. 
a abandonar sus islas. Ante tal situación, la vida en Kiribati es gradualmente insegura, por lo que el señor Teitiota pidió protección, en principio para él, pero posteriormente también para su familia, a través del otorgamiento del estatus de refugiado, alegando que su vida, la de su mujer y las de sus tres hijos, nacidos en Nueva Zelanda, corrían peligro en Kiribati.

Un oficial de la Unidad de Refugiados y Protección rechazó su solicitud, la cual fue apelada en primera instancia ante el Tribunal de Inmigración y Protección, a pesar de que el Tribunal aceptó el argumento del señor Teitiota desestimó su apelación, sosteniendo que él "no es considerado un refugiado en los términos de la Convención de Ginebra sobre el Estatuto de los Refugiados de 1951, ni una persona protegida de conformidad con el Pacto Internacional de Derechos Civiles y Políticos". Con posterioridad se presentaron múltiples apelaciones ante la Corte de Apelación y la Corte Superior.

\section{Resolución final}

La resolución final fue emitida el 20 de julio de 2015 por la Corte Suprema de Nueva Zelanda, en donde se analizaron las situaciones de derecho presentadas ante las cortes anteriores, las cuales en esencia fueron las siguientes:

1) Si de conformidad con la Immigration Act 2009 se extiende la protección a quien enfrenta desplazamiento por motivos ambientales.

2) Si deben implementarse convenios internacionales con relación al cuidado de los tres hijos del señor Teitiota, menores de seis años nacidos en Nueva Zelanda.

Respecto a la primera cuestión, en el ámbito del derecho internacional público se planteó si es aplicable la protección al ciudadano de Kiribati de acuerdo con el artículo 1o., apartado A, 2) de la Convención de Ginebra de 1951; lo cual a su vez se debía relacionar con la manera en que dicho artículo es incorporado en la legislación interna de Nueva Zelanda, como una base para la interpretación del término de refugiado en la sección 129 de la Immigration Act 2009. 
En cuanto a la segunda, se planteó si la Convención de las Naciones Unidas sobre los Derechos del Niño es relevante para la evaluación del daño para los fines de la Convención de Ginebra; y si el derecho a la vida de acuerdo con el Pacto Internacional de Derechos Civiles y Políticos implica el derecho de un pueblo a no ser privado de sus medios de subsistencia.

La resolución finalmente ratificó las decisiones tanto del Tribunal de Inmigración y Protección, así como la de la Corte de Apelación y la Corte Superior, cuyos argumentos a destacar en este breve estudio, especialmente en cuanto a la aplicación de la Convención de Ginebra sobre el Estatuto de los Refugiados de 1951, son los siguientes:

En principio, el término de refugiado no puede entenderse únicamente como el establecido por la Convención, por lo que una persona puede ser llamada refugiada por los impactos del cambio climático, sin embargo, esta causa no es contemplada en la definición legal. Tampoco se establece un mecanismo para el reasentamiento internacional de personas que se encuentran en la situación del señor Teitiota.

Especialmente, se puntualizó sobre el concepto de "ser perseguido", el cual no es definido en la Convención de Ginebra ni en su Protocolo de Nueva York, adoptado en 1967, sin embargo, el Tribunal de Inmigración y Protección de Nueva Zelanda aplica el siguiente concepto: "la violación sostenida y sistemática de derechos humanos como consecuencia de un fallo de protección estatal", ${ }^{65}$ de tal manera que el riesgo de ser perseguido debe ser bien fundado, en el sentido de ser real y no especulativo.

En este caso, el abogado del señor Teitiota planteó el "acto de huir del cambio climático" debido al daño grave que se causará a su defendido y a su familia junto con la falta de voluntad o incapacidad del gobierno de Kiribati para lidiar con los impactos del cambio climático. Asimismo, sostuvo que la palabra "refugiado" puede abarcar a las personas que tienen que huir independientemente de la causa. Como apoyo, citó la etimología latina de la palabra "perseguir" que, según él, tiene una "voz pasiva de huir de algo o una cualidad activa de seguir a alguien”. En este caso, señaló que la persecución no requiere un actor en el sentido pasivo, como lo es el "acto de huir del cambio climático". ${ }^{66}$

65 Véase Refugee Appeal No. 74665, New Zealand, Refugee Status Appeals Authority, 7 July 2004.

66 AF (Kiribati) [2013] NZIPT 800413, párr. 51. 
Ante lo cual, el Tribunal de Inmigración y Protección se basó en un estudio de Astri Suhkre, ${ }^{67}$ quien distingue entre una "concepción sociológica" de refugiado y una legal donde la primera es más amplia que la segunda. No obstante, tanto el derecho internacional como el interno sólo permiten el reconocimiento como refugiado si los requisitos de la Convención se cumplen, es decir, que exista una persecución por alguna de las cinco causas estipuladas. Se aplica la concepción legal mas no la sociológica, donde quien es legalmente considerado como refugiado está huyendo de su propio gobierno o de un actor no estatal, del cual el gobierno no quiere o no puede protegerlo.

En este caso, tanto el Tribunal de Inmigración y Protección como la Corte de Apelación no encontraron una posibilidad real de que el señor Teitiota sea perseguido individualmente si es devuelto a Kiribati, determinó que no hay pruebas de que él enfrente una posibilidad real de sufrir daños físicos graves a causa de la violencia relacionada con las disputas por la vivienda, la tierra y la propiedad en Kiribati en el futuro; tampoco hay pruebas de que las condiciones ambientales a las que se enfrentó el solicitante o que probablemente enfrentaría al regresar a Kiribati pongan en peligro su vida y la de su familia, impidiéndoles reanudar su vida de subsistencia con dignidad; es decir, al regresar a su tierra natal, no hay evidencias de que sufriría una violación sostenida y sistémica de sus derechos humanos básicos, como el derecho a la vida en virtud del artículo 6o. del Pacto Internacional de Derechos Civiles y Políticos (PIDCP) o el derecho a una alimentación, vestimenta y vivienda adecuadas en virtud del artículo 11 del Pacto Internacional de Derechos Económicos, Sociales y Culturales (PIDESC). Si bien es cierto que Kiribati enfrenta grandes desafíos, la Corte señaló que no hay pruebas de que el gobierno de este país no está tomando acciones, en la medida de lo posible, para proteger a sus ciudadanos de los efectos de la degradación ambiental por el cambio climático, donde la posición del señor Teitiota no parece ser diferente a la de cualquier otro nacional de Kiribati. ${ }^{68}$

En concreto la Corte de Apelación resolvió que "los efectos del cambio climático no son una causa incluida en la Convención de Refugiados, por lo que la aplicación de este instrumento legal no es la solución al problema

67 Ibidem, párrs. 52 y 53.

68 Ibidem, párrs. 72-74; Ioane Teitiota vs. The Chief Executive of the Ministry of Business, Innovation and Employment [2014] NZCA 173, párrs. 21-29. 
de Kiribati”; ${ }^{69}$ lo cual es ratificado en la decisión final de la Suprema Corte de Nueva Zelanda.

Sin embargo, es importante hacer énfasis que en el ámbito temporal de validez estudiado, el asunto fue infundado, pero deja abierta la posibilidad de que en circunstancias fácticas diferentes el resultado jurídico sea otro; lo cual se desprende de lo expuesto por el Tribunal y las cortes de Apelación y Superior, en el argumento siguiente: "their decisions did not mean that environmental degradation resulting from climate change or other natural disasters could never create a pathway into the Refugee Convention or protected person jurisdiction”. Secundado por la Suprema Corte al señalar que: "Our decision in this case should not be taken as ruling out that possibility in an appropriate case". ${ }^{70}$ Por lo tanto, es un precedente no taxativo que abre la posibilidad de reinterpretar la Convención en otros casos, o bien, propiciar su enmienda, que en su caso requerirá del consenso internacional de los Estados Parte; ahora bien, por lo que toca al ámbito interno, quizás sea propicia una interpretación más amplia de la Convención al momento de adoptarla en el régimen nacional.

\section{REFLEXIÓN FINAL}

Las migraciones por causas ambientales o vinculadas a factores ambientales se han generado a lo largo de la historia; no obstante, actualmente se prevé un mayor movimiento poblacional a consecuencia de los impactos del cambio climático antropogénico, donde el desplazamiento puede ser interno o transfronterizo, con especial repercusión, en este último caso, debido a la alta vulnerabilidad de los pequeños Estados insulares a los impactos del cambio climático, cuyo principal riesgo es la elevación del nivel del mar, observada y proyectada, que propiciará la desaparición de estos Estados, por lo que sus poblaciones requerirán protección jurídica internacional

69 Ioane Teitiota vs. The Chief Executive of the Ministry of Business, Innovation and Employment [2014] NZCA 173, párr. 21.

70 Ioane Teitiota vs. The Chief Executive of the Ministry of Business, Innovation and Employment, [2015] NZSC 107, párr. 13; Ioane Teitiota vs. The Chief Executive of the Ministry of Business, Innovation and Employment [2014] NZCA 173, párr. 18; AF (Kiribati) [2013] NZIPT 800413, párr. 55. 
que les asegure una reubicación planificada fuera de sus países de origen que garantice el respeto de sus derechos.

El papel que se le atribuye a los Estados es fundamental ante la responsabilidad de afrontar este tipo de desplazamientos, en el caso de una migración interna, la responsabilidad es, principalmente, de cada gobierno y el asunto es meramente interno, mientras que cuando se tienen que cruzar fronteras es aplicable el marco jurídico internacional, vinculado con el alcance de las políticas nacionales en materia de migración.

En este contexto, aquellos que migran internacionalmente debido a los impactos del cambio climático, difícilmente pueden obtener protección de conformidad con lo dispuesto en la Convención de Ginebra sobre el Estatuto de los Refugiados de 1951, ya que esas causas no son expresamente reconocidas para obtener el estatus de refugiado.

En este sentido, el caso del señor Teitiota es indicativo, ello a pesar de haberse destacado las dificultades que Kiribati enfrenta con respecto a los impactos del cambio climático, especialmente por la elevación del nivel del mar y el consecuente daño costero por la erosión; las pérdidas de infraestructura; la reducción de la calidad y cantidad de los recursos hídricos; los daños a la agricultura; y el deterioro de la salud pública. Simplemente, tales circunstancias no fueron suficientes para la Suprema Corte de Nueva Zelanda a efecto de dar cabida a una interpretación legal de la Convención, ya que ésta define al refugiado delimitando los requisitos para obtener ese estatus, los cuales no están estipulados para reconocer y dar protección a los migrantes por el cambio climático. Por ahora, la Convención de Ginebra de 1951 no constituye el instrumento jurídico aplicable, a menos que, en atención a la naturaleza dinámica y evolutiva del derecho, ésta fuese enmendada por consenso de los Estados parte para atender uno de los grandes impactos sociales del cambio climático como son los movimientos poblacionales, una realidad que hoy gira en torno a ausencias legales en el contexto internacional. Su inclusión en la Convención sería lo idóneo, sin embargo, el cambio climático proyecta impactos sin precedentes, cuyas consecuencias son complejas y con repercusiones sociales a gran escala que deben ser tratadas de manera particular con el objetivo de buscar una mayor y eficaz protección.

De tal manera que habrá de actuarse con relación a dos grandes posibilidades: la primera, el reconocimiento del migrante por cambio climático mediante un concepto legal e unívoco en el ámbito del derecho internacional que permita definir con claridad el instrumento jurídico aplicable, entre 
Esta revista forma parte del acervo de la Biblioteca Jurídica Virtual del Instituto de Investigaciones Jurídicas de la UNAM

los existentes; o bien, como segunda opción crear una nuevo instrumento que además de otorgar certidumbre conceptual, en paralelo estructure una regulación específica. Uno u otro camino dependerá del alcance en la protección y de la responsabilidad que los Estados estén dispuestos a asumir.

\section{BIBLIOGRAFÍA}

Climate Change, About Kiribati, disponible en http://www.climate.gov.ki/ about-kiribati/.

Biermann, Frank y BOAS Ingrid, "Climate Change and Human Migration. Towards a Global Governance System to Protect Climate Refugees”, en SChefFran, J. et al. (eds.), Climate Change, Human Security andViolent Conflict, Berlin, Springer-Verlag, 2012.

BLACK, Richard, "Environmental Refugees: Myth or Reality?", New Issues in Refugee Research, Working Paper 34, UNHCR, 2001.

BOANO, C. et al., Environmentally Displaced People Understanding the Linkages between Environmental Change, Livelihoods and Forced Migration, Oxford, U. K., Refugee Studies Centre, Oxford Department of International Development, University of Oxford, 2008.

BOrRÁs, Susana, "Refugiados ambientales: el nuevo desafío del derecho internacional del medio ambiente”, Revista de Derecho, vol. XIX, núm. 2, diciembre de 2006.

BRONEN, Robin, "Las comunidades de Alaska: derechos y resistencia”, Revista Migraciones Forzadas, núm. 31, 2008.

Brown, Oli, "El baile de cifras", Revista Migraciones Forzadas, núm. 31, 2008. Changing Climate or Kiribati, disponible en: http: / /www.climate.gov. ki/changing-climatel.

División de Estadística, DePARTAMENTO DE InFormación, ECONÓmiCA Y SOCIAl y ANÁlisis De POlíticas, Glosario de Estadísticas del Medio Ambiente, Nueva York, Naciones Unidas, 1997.

Droege, Cordula, "Progresos en la protección jurídica de los desplazados internos", Revista Migraciones Forzadas GP 10, diciembre de 2018.

Dun, Olivia y GEMENNE, François, "Definir la migración por motivos medioambientales”, Revista Migraciones Forzadas, núm. 31, 2008. 
Esta revista forma parte del acervo de la Biblioteca Jurídica Virtual del Instituto de Investigaciones Jurídicas de la UNAM

EDWARDS, Adrian, “¿«Refugiado» o «migrante»? ¿Cuál es el término correcto? Los dos términos tienen diferentes significados, y confundirlos conduce a problemas para ambas poblaciones", UNHCR. ACNUR La Agencia de la ONU para los Refugiados, 11 de julio de 2016, disponible en www. acnur.org/noticias/noticia/2016/7/5b9008e74/refugiado-o-migrante-cuales-el-termino-correcto.html.

EGEA, Carmen y SOLEDAD, Javier Iván, "Los desplazados ambientales, más allá del cambio climático. Un debate abierto”, Cuadernos Geográficos, núm. 49, 2011.

EL-Hinnawi, Essam, Environmental Refugees, Nairobi, Kenya, United Nations Environment Programme (UNEP), 1985.

ENVIRONMENT AND CONSERVATION DIVISION, MinisTRY OF ENVIRONMENT, Land, And Agricultural Development Government of Kiribati, Republic of Kiribati. National Adaptation Programme of Action (NAPA), 2007.

FAO, Climate Change: Implications for Food Safety, Food and Agriculture Organization of the United Nations, 2008.

FMO, "What is Forced Migration?", en http://www.forcedmigration.org/ about/whatisfm, 2012.

"Future climate of Kiribati", disponible en: http: / / www.climate.gov.ki / changing-climatel.

Global Protection Cluster, Marcos normativos sobre desplazamiento interno. Desarrollos mundiales, regionales y nacionales, The UN Refugee Agency (UNHCR), Internal Displacement Monitoring Centre (IDMC), Norwegian Refugee Council (NRC), 2016.

Graeme, Hugo, "Environmental Concerns and International Migration", The International Migration Review, vol.30, no. 1, Special Issue: Ethics, Migration, and Global Stewardship, primavera de 1996.

IBARRA, Rosalía, "Cambio climático y conflictos socioambientales: Migraciones forzadas", en CARMONA LARA, María del Carmen Aurora y ACUÑa HeRnÁNDEZ, Ana Laura, La Constitución y los derechos ambientales, México, UNAM, Instituto de Investigaciones Jurídicas, 2015.

IBARRA, Rosalía, "Cambio climático, seguridad alimentaria y el derecho humano a la alimentación”, en IBARRA, Rosalía (coord.), Cambio climático y gobernanza. Una visión transdisciplinaria, México, UNAM, Instituto de Investigaciones Jurídicas, 2019 (en prensa). 
Esta revista forma parte del acervo de la Biblioteca Jurídica Virtual del Instituto de Investigaciones Jurídicas de la UNAM

IDMC y NRC, Displacement Due to Natural Hazard-Induced Disasters, Ginebra, Internal Displacement Monitoring Centre, Norwegian Refugee Council (NRC), 2011.

IDMC y NRC, Global Estimates 2012: People Displaced by Disasters, Ginebra, Internal Displacement Monitoring Centre, Norwegian Refugee Council (NRC), 2012.

IDMC y NRC, Global Report on Internal Displacement GRID 2018, Ginebra, Internal Displacement Monitoring Centre, Norwegian Refugee Council (NRC), 2018.

IDMC, NRC y UN OCHA, Monitoring disaster displacement in the context of climate change, Ginebra, Internal Displacement Monitoring Centre (IDMC), Norwegian Refugee Council, United Nations Office for the Coordination of Humanitarian Affairs, 2009.

IOM, Glossary on Migration, 2a. ed., International Migration Law, núm. 25, Ginebra, International Organization for Migration, 2011.

IPCC, "Resumen para responsables de políticas", en FIELD, C. B., et al. (eds.), Cambio climático 2014: Impactos, adaptación y vulnerabilidad. Contribución del Grupo de trabajo II al Quinto Informe de Evaluación del Grupo Intergubernamental de Expertos sobre el Cambio Climático, Ginebra, Organización Meteorológica Mundial, 2014.

Kelly WyetT, "Escaping a Rising Tide: Sea Level Rise and Migration in Kiribati”, Asian \&The Pacific Policy Studies, vol. 1, issue 1, 2013.

Lonergan, Steve, "The Role of Environmental Degradation in Population Displacement", Environmental Change and Security Program Report, Issue 4, primavera de 1998.

Ministry of Internal \& Social Affairs, 6. South Tarawa, Republic of Kiribati Island Report Series, Office of Te Beretitenti and T'Makei Services, 2012, disponible en: http://www.climate.gov.ki/wp-content/ uploads/2013/01/6_SOUTH-TARAWA-revised-2012.pdf.

Miraglia, M. et al., "Climate Change and Food Safety: An Emerging Issue with Special Focus on Europe”, Food and Chemical Toxicology, núm. 47, 2009.

MYERS, Norman, Enviromental Refugees: An Emergent Security Issue, 13th Meeting of the OSCE Economic Forum, Session III - Environment and Migration, Organization for Security and Cooperation in Europe, Praga, 23-27 de mayo de 2005. 
Esta revista forma parte del acervo de la Biblioteca Jurídica Virtual del Instituto de Investigaciones Jurídicas de la UNAM

NeWland, Kathleen, Climate Change and Migration Dynamics, Washington, D.C., USA, Migration Policy Institute, 2011.

OIM, Diálogo Internacional sobre la Migración. Seminario de Expertos: Migración y Medio Ambiente, núm. 10, Ginebra, Organización Internacional para las Migraciones y Programa de Políticas e Investigación sobre Migraciones, 2008.

OIM, Migración y cambio climático, Ginebra, Organización Internacional para las Migraciones, Serie de Estudios de la OIM sobre la Migración, núm. 31, 2008.

OIM, Informe sobre las Migraciones en el Mundo 2010. El futuro de la migración: creación de capacidades para el cambio, Ginebra, Organización Internacional para las Migraciones, 2010.

OIM, Medioambiente, cambio climático y migración: perspectiva y actividades de la OIM, Ginebra, Organización Internacional para las Migraciones, 2011.

OIM, Informe sobre las migraciones en el mundo 2018, Ginebra, Organización Internacional para las Migraciones, 2018.

OPS, Proteger la salud frente al cambio climático: evaluación de la vulnerabilidad y la adaptación, Washington, USA, Organización Panamericana de la Salud, 2012.

ORSI, Adriana, “Desplazados ambientales” Futuros, vol. VI, no. 20, 2008.

OSWALD-SPRING, Úrsula et al., Vulnerabilidad social y género entre migrantes ambientales, México, UNAM, Centro Regional de Investigaciones Multidisciplinarias, 2014.

Richmond, A. H., "The Environment and Refugees: Theoretical and Policy Issues”, Population Bulletin of the United Nations, núm. 39, United Nations, Nueva York, 1995.

Robles, Agustín y Garatuza, Jaime, "Cambio climático en México: impactos esperados en la disponibilidad del agua”, en CRuz, Xóchitl et al. (coords.), México ante la urgencia climática: ciencia, política y sociedad, México, UNAM, CRIM, CIICH, PICC, 2015.

SÁNCHEZ COHEN, Ignacio et al., "Impacto de la variabilidad climática en la disponibilidad de agua para producción agrícola en México", en CRUZ, Xóchitl et al. (coords.), México ante la urgencia climática: ciencia, política y sociedad, México, UNAM, CRIM, CIICH, PICC, 2015.

Soledad, Javier Iván, Desplazamiento interno en Colombia. La situación de los desplazados en El Catatumbo, España, Editorial Universidad de Granada, 2009. 
Esta revista forma parte del acervo de la Biblioteca Jurídica Virtual del Instituto de Investigaciones Jurídicas de la UNAM

STATISTICS Division, DEPARTMENT FOR ECONOMIC AND SOCIAL INFORMATION AND POlicy ANAlysis, Glossary of Environment Statistics, New York, United Nations, 1997.

Woods, William B., "Ecomigration. Linkages Between Environmental Change and Migration”, en Zolberg, Aristide R. y Benda, Peter M. (eds.), Global Migrants Global Refugees. Problems and Solutions, New YorkOxford, Berghahn Books, 2001. 